\title{
Detection and Classification of Haze Affected Images Using CNN Approach
}

\author{
SenthilKumar K Pa, Sivakumar $\mathbf{P}^{\mathrm{b}}$ and MohanasundariL ${ }^{\mathrm{c}}$ \\ Assistant professor, Department of ECE, Easwari Engineering College, Chennai, \\ India \\ bProfessor, Department of ECE, Dr.N.G.P Institute of Technology, Coimbatore, India \\ 'Assistant professor, Department of ECE, Saveetha Engineering College, Chennai, \\ India.
}

Article History: Received: 11 January 2021; Accepted: 27 February 2021; Published online: 5 April 2021

\begin{abstract}
Detection and classifications of the haze affected image is important for the real time multimedia data transmission and reception in remote mode in order to improve the quality of the received image or video sequences. In this paper, Convolutional Neural Networks (CNN) classification approach is used with Shearlet Transform for the detection and segmentation of haze affected images.The image to be tested for haze pattern detection is preprocessed and then it is decomposed with shearlet transform. The features are computed from the shearlet transform decomposed coefficients and then these computed features are classified by the deep learning CNN for identifying the haze affected images. This proposed haze classification method is tested on both indoor and outdoor environmental images.
\end{abstract}

Keywords: Haze, classification, shearlet transform, CNN, quality

\section{Introduction}

Today modern and advanced world requires fast accessible real time devices for transferring and receiving multimedia applications from one location to another location in remote manner. This requires error free data transmission and reception with respect to various data types and band width modes. Due to the improper weather conditions, the transmitted or received video or image quality is degraded. Most of the real time multimedia data transmission has been affected by haze, fog and rain water particles and dust particles also. The received sensor in remote environment receives the transmitted light energy along with the radiation pattern of the affected unwanted components like dust, haze and fog [12-15]. Hence, there is a need for detecting and removing such unwanted components from the received video or image sequences in order to improve the quality of the received content. The distortion of color in image causes haze pixels which degrades the quality of the original transmitted image or video sequences. In this paper, the haze affected image is detected and classified before the haze removal process starts. Most of the conventional algorithms are directly applied on all type of images which does not consider whether the image is affected by haze or not. These algorithms destroyed the pixel quality if the image is not affected by the haze. Hence, the haze image classification is considered as most important to improve the quality of the received video or image sequences.

Fig. 1 (a) shows the haze affected outdoor image and Fig.1 (b) shows the haze affected indoor image.

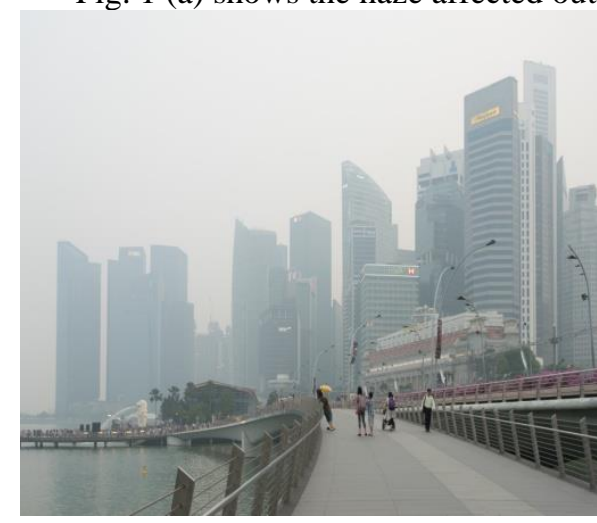

(a)

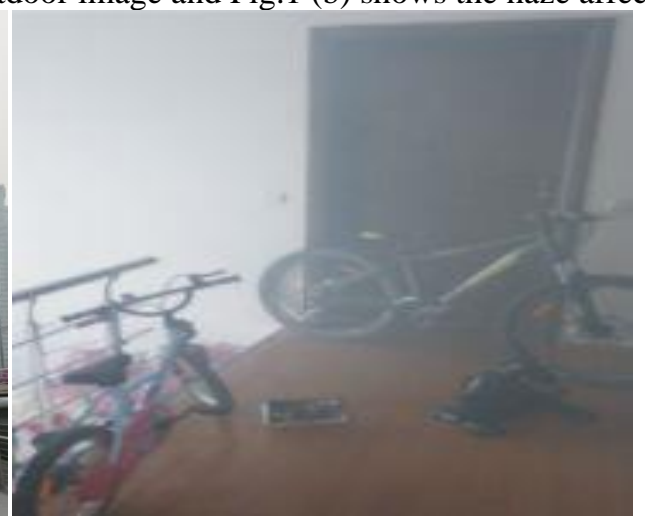

(b)

Figure 1 (a) Haze affected outdoor image (b) Haze affected indoor image

\section{Literature survey}

Hassan et al. (2020)used guidance filter by detecting and segmenting super pixels on the haze affected image. The parameters of the proposed guidance filter were set by the supervised method. The authors tested this guidance filter based haze image detection and classification approach on both indoor and outdoor environmental images to validate the simulation results with respect to robustness property. The authors obtained $97.1 \%$ of Se, $97.6 \%$ of Sp, and $97.6 \%$ of Acc on indoor set of images and also they obtained $94.7 \%$ of Se, $95.2 \%$ of Sp, and $96.1 \%$ of Acc on indoor set of images. Kang et al. (2020) proposed dehazing model approach usingtotal variation 
scheme and inter-channel correlation property for identifying the haze affected pixels in the real time images. This method used non-linear behavior of the super pixels for differentiating the haze affected pixels from the normal pixels in both indoor and outdoor environmental images. The authors obtained $96.5 \%$ of Se, $97.1 \%$ of Sp, and $97.2 \%$ of Acc on indoor set of images and also they obtained $95.9 \%$ of Se, $95.3 \%$ of Sp, and $96.8 \%$ of Acc on indoor set of images. Guo et al. (2019)used HSI Color Space model for detecting and removing the haze pixels in both indoor and outdoor environmental images. The color space model generated the pixel invariant model for obtaining the non-linear behavior in order to remove the haze pixels completely from the single source images. The authors applied their developed haze image detection and classification approach on the remote sensing images and they obtained $96.1 \%$ of Se, $98.1 \%$ of $\mathrm{Sp}$, and $98.3 \%$ of Acc on indoor set of images and also they obtained $94.1 \%$ of Se, $95.3 \%$ of Sp, and $96.4 \%$ of Acc on indoor set of images.

Tian et al. (2016) used dark channel prior method for detecting the haze super pixels from the single source image. The authors derived the matrix scheme for locating the haze pixels in the single source image through the identification of the super pixel behavior of the haze. This method was tested on different set of real time environmental images to evaluate the performance of the haze image detection and classification.Kim et al. (2013) developed haze pixel detection scheme using optimized contrast enhancement approach. This method separated every frame from the real time video and the separated sequences were tested for haze pixel detection. The haze pixels were not properly enhanced by this approach and the developed model was applied on the real time environmental images.

\section{Proposed methodologies}

In this article, the images are classified into haze affected image or non-haze affected image using deep learning architecture. The image to be tested for haze pattern detection is preprocessed and then it is decomposed with shearlet transform. The features are computed from the shearlet transform decomposed coefficients and then these computed features are classified by the deep learning Convolutional Neural Networks. The generic work flow of the proposed haze image classification is illustrated in Fig.2.

Fig. 2 (a) is the haze and non-haze pattern image training and Fig.2 (b) is the haze image classification by proposed method.

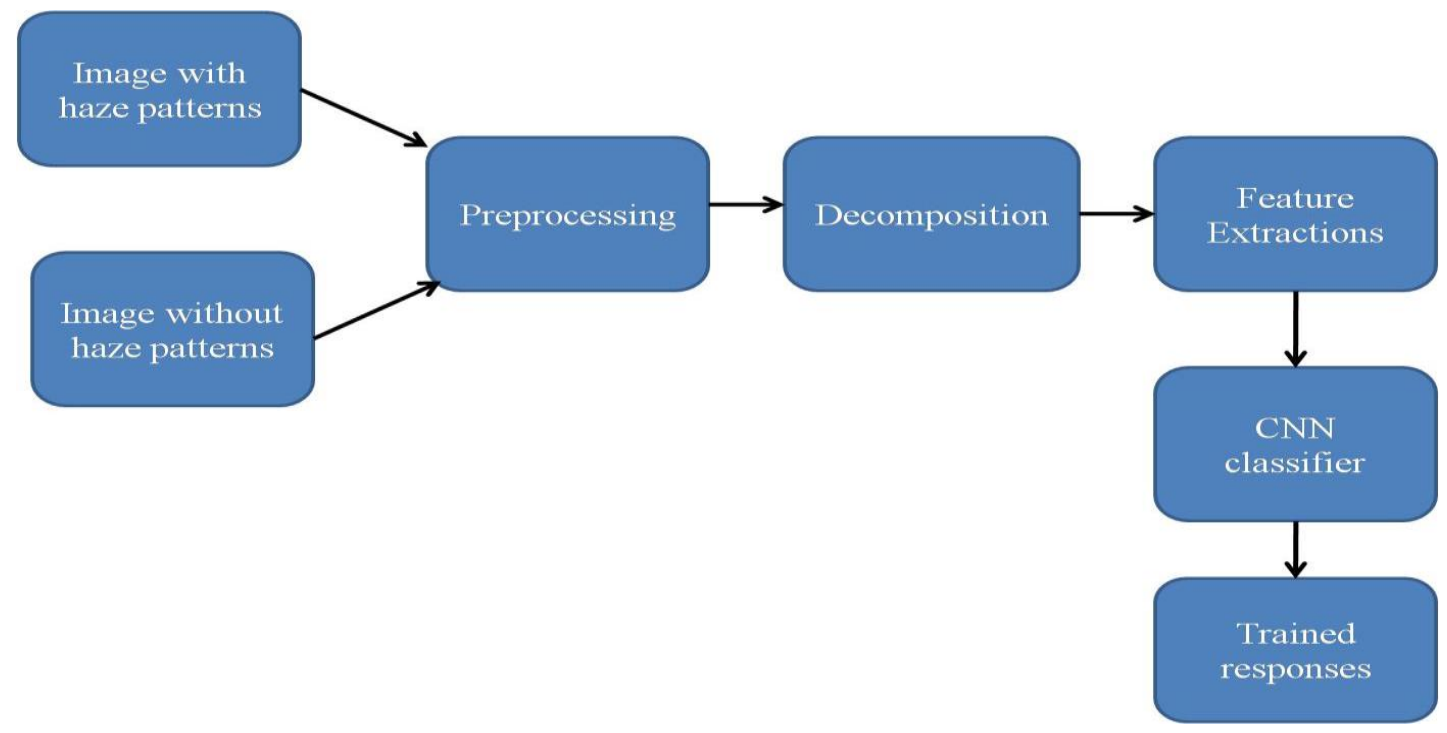

(a)

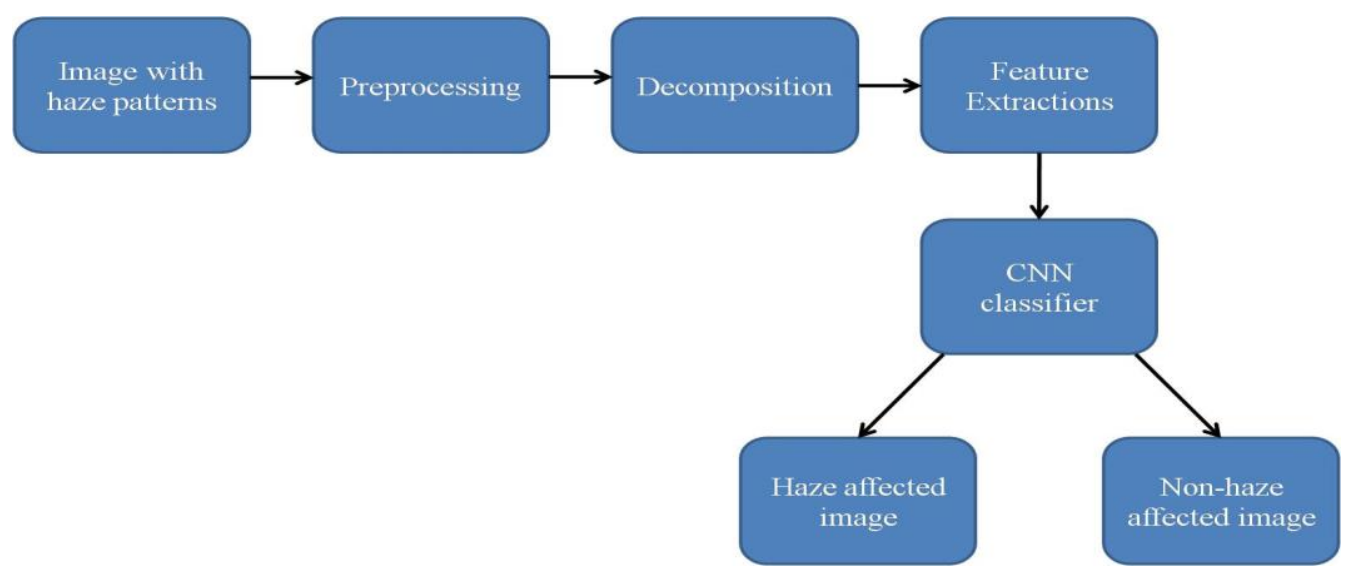

(b)

Figure 2 (a) Haze and non-haze pattern image training (b) Haze image classification by proposed method 


\section{Preprocessing}

The images are in RGB format where each pixel contains 24 bits. Hence, the processing of all pixels for haze image classification is quite complex in terms of detection time. Hence, the RGB image is converted into luminance-chrominance pattern in this paper. Then, the luminance image is processed further to detect the haze affected image. The conversion of the RGB image into luminance-chrominance pattern image is depicted with the following equation.

$$
\begin{aligned}
& \text { Luminance }=16+\frac{65.73 R}{256}+\frac{129.05 G}{256}+\frac{25.06 B}{256} \\
& \text { Chrominance }_{b}=1128-\frac{37.94 R}{256}-\frac{74.49 G}{256}+\frac{112.436 B}{256} \\
& \text { Chrominance }_{r}=128+\frac{112.43 R}{256}-\frac{94.15 G}{256}-\frac{18.28 B}{256}
\end{aligned}
$$

Each pixel constitutes Red (R), Green $(\mathrm{G})$ and Blue (B) pattern and the luminance image is derived by substitute $\mathrm{R}, \mathrm{G}$ and $\mathrm{B}$ pixel pattern in the above conversion equation.

\section{Shearlet Transform}

The multi scale and multi directionality of the image can be achieved using the shearlet transform in this article (Liang Ding et al. 2015). The applications of the shearlet transform includes image processing and signal processing with respect to object detection, edge detection and image restoration problems (Gibert et al. 2014). In this article, the shearlet transform is used for object (haze) detection in image processing applications.

The shearlet transform consists of low pass and high pass filters. The transfer function of the low pass filter is illustrated by $\mathrm{H} 1 \mathrm{z}$ ) and the transfer function of the high pass filter is illustrated by H0 (z), respectively. At each stage, the luminance image is decomposed using the constructed low pass and high pass filter transfer functions as shown in the figure. The sub band 1 is obtained by convolving the low pass filter response with the kernel of directional filtering at stage 1 . The sub band 2 is obtained by convolving the low pass filter response with the kernel of directional filtering at stage 2 . The sub band 3 is obtained by convolving the low pass filter response with the kernel of directional filtering at stage 3 . The sub band 4 is obtained by convolving the high pass filter response with the kernel of directional filtering at stage 4.

Figure 3 shows the decomposition of luminance image into four numbers of sub bands using shearlet transform.

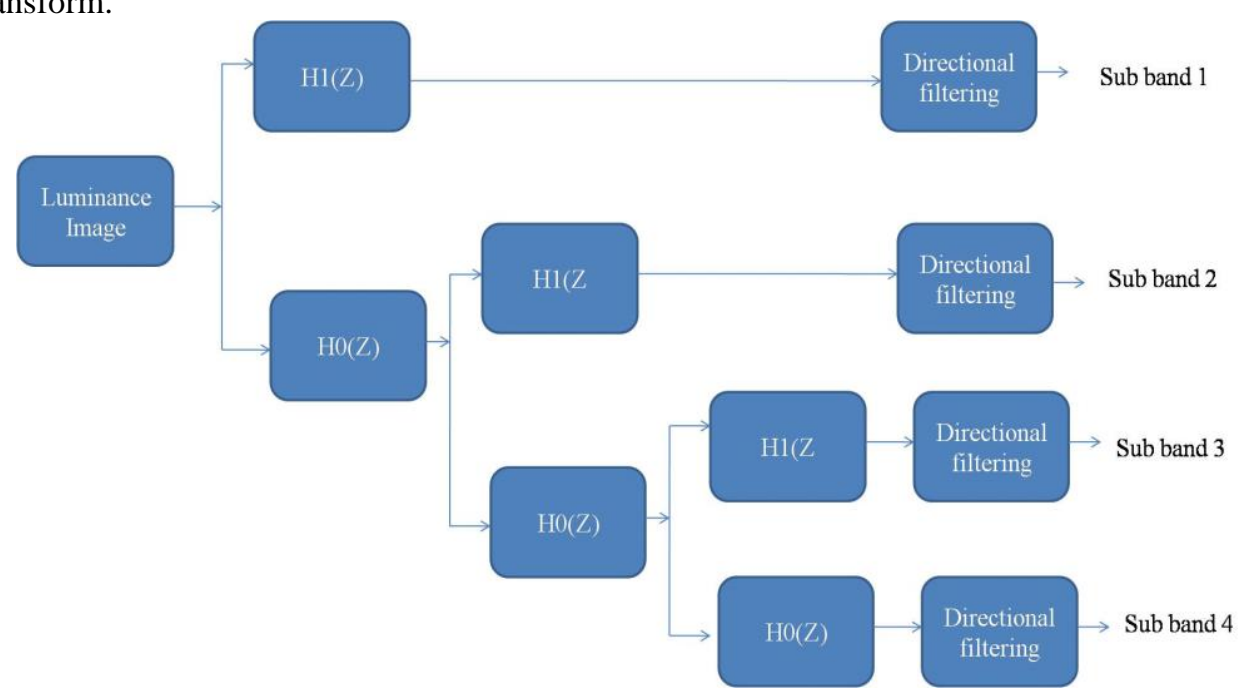

Figure 3 Decomposition of luminance image using shearlet transform

Feature Extractions

The decomposed sub bands from the shearlet transform are represented as $s 1(i, j), s 2(i, j), s 3(i, j)$ and $s 4(i, j)$.The decomposed coefficients are stored in matrix format in this decomposed sub band with respect to row and column. From these decomposed sub bands, the features as Shearlet Index Feature (SIF), Shearlet Metric Feature (SMF), Shearlet Kappa Feature (SKF) and Shearlet Basis Feature (SBF) are computed using the following equations.

Shearlet Index Feature $=\frac{1}{M * N} \frac{\sum_{i=1}^{M-1} \sum_{j=1}^{N-1} S 1(i, j)}{\sum_{i=1}^{M-1} \sum_{j=1}^{N-1} S 2(i, j)}$

Where, $\mathrm{M}$ and $\mathrm{N}$ are the rows and columns of the decomposed sub band by shearlet transform.

$$
\begin{aligned}
& \text { Shearlet Metric Feature }=\frac{1}{M * N} \frac{\sum_{i=1}^{M-1} \sum_{j=1}^{N-1}[S 1(i, j)+S 2(i, j)]}{\sum_{i=1}^{M-1} \sum_{j=1}^{N-1}[S 3(i, j)+S 4(i, j)]} \\
& \text { ShearletKappa Feature }=\frac{1}{M * N} \frac{\sum_{i=1}^{M-1} \sum_{j=1}^{N-1} S 1(i, j)}{\sum_{i=1}^{M-1} \sum_{j=1}^{N-1}[S 2(i, j)+S 4(i, j)]} \\
& \text { Shearlet Basis Feature }=\frac{1}{M * N} \frac{\sum_{i=1}^{M-1} \sum_{j=1}^{N-1} S 2(i, j){ }^{2}}{\sum_{i=1}^{M-1} \sum_{j=1}^{N-1}[S 1(i, j)+S 2(i, j)+S 3(i, j)]^{2}}
\end{aligned}
$$

All these computed features are combined in a single matrix and they are fed into the classifier which is explained in the following section. 


\section{Classifications}

The classifier is used to classify the objects or patterns in an image using the feature computed values. In this article, $\mathrm{CNN}$ is used to classify the test image into either haze affected image or non-haze affected image. The proposed CNN architecture used in this article is designed with Convolutional layer, pooling layers and feed forward Neural Networks (NN) layers. The designed CNN is constituted with 3 Convolutional layers and each Convolutional layer is followed by pooling layer (Max-polling algorirthm). The aim of this pooling layer is to reduce the size of the responses from each Convolutional layer. The final response from the final pooling layer is fed into the NN which is designed with single input, single output and 10 numbers of hidden layers. Each hidden layer is designed with 15 numbers of neurons and the neurons in input and output layer are chosen based on the extracted features in this article. The NN layer in proposed CNN architecture produces index value. The index value is negative for the haze affected image and the index value is positive for the non-haze affected image.

Fig. 4 is the classification of haze affected images using CNN classification approach.

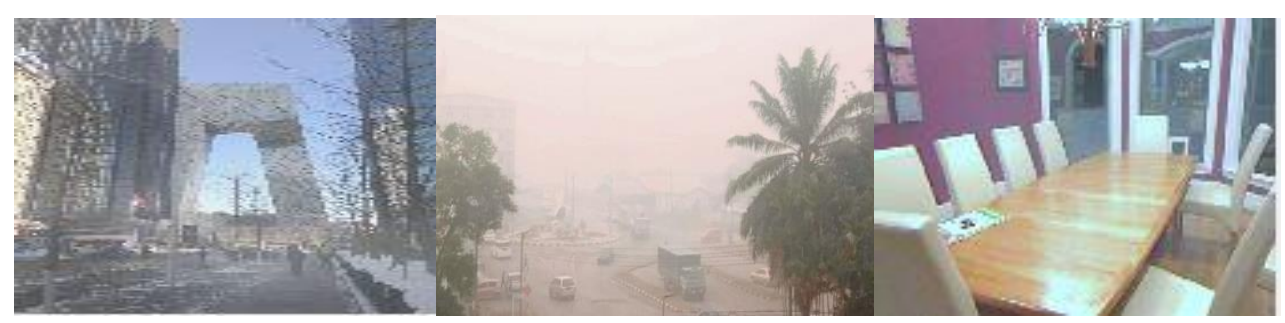

Figure 4 Haze detected and classified images using CNN approach

\section{Results and Discussions}

In this article, MATLAB R2018 is the application software used which was designed by Mathworks Company for simulating the proposed haze image classifications.Fig. 5 is the confusion matrixes for haze image classifications which can be described by the performance evaluation parameters Sensitivity (Se), Specificity (Sp), Accuracy (Acc), Positive Predictive Value (PPV) and Negative Predictive Value (NPV), respectively. The 'True Positive' describes the correctly detected haze image with respect to positive in confusion matrix and 'False Negative' describes the correctly detected haze image with respect to negative. The 'False Positive' describes the correctly detected 'No haze image' with respect to positive in confusion matrix and 'True Negative' describes the correctly detected 'No haze image' with respect to negative. These values are computed based on the ground truth images which are available in open access dataset.

$$
\text { Haze present No Haze present }
$$

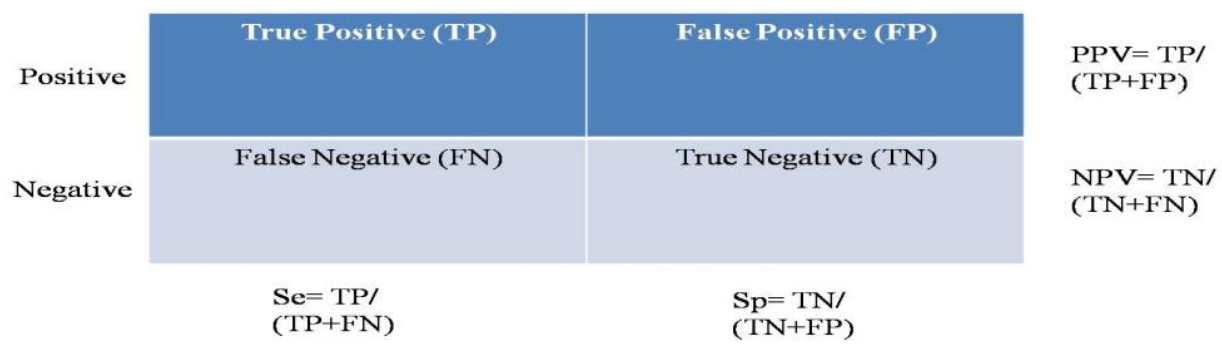

$\mathrm{Acc}=(\mathrm{TP}+\mathrm{TN}) /$

$(\mathrm{TP}+\mathrm{FP}+\mathrm{TN}+\mathrm{FN})$

\section{Figure 5 Confusion matrixes for haze image classifications}

Table 1 is the simulation results analysis of the proposed haze image classifications on Indoor images.

Table 1 is the simulation results analysis of the proposed haze image classifications on indoor images. The proposed haze image classification system obtains $98.3 \%$ of sensitivity, $98.56 \%$ of specificity, and $99.1 \%$ of accuracy, $98.14 \%$ of PPV and $98.2 \%$ of NPV.

Table 1 Simulation results analysis of the proposed haze image classifications on Indoor images

\begin{tabular}{|c|c|c|c|c|c|}
\hline \multirow{2}{*}{$\begin{array}{c}\text { Image } \\
\text { sequences }\end{array}$} & \multicolumn{2}{|c|}{ Performance evaluation parameters in \% } & PPV & NPV \\
\cline { 2 - 6 } & Se & Sp & Acc & 97.1 & 98.4 \\
\hline 1 & 97.7 & 98.1 & 98.5 & 98.6 & 98.5 \\
\hline 2 & 98.1 & 98.7 & 98.6 & 98.9 & 98.9 \\
\hline 3 & 98.6 & 98.5 & 99.6 & 98.3 & 98.2 \\
\hline 4 & 97.7 & 98.9 & 98.5 & 97.7 & 99.6 \\
\hline 5 & 98.5 & 99.1 & & & 9 \\
\hline
\end{tabular}


SenthilKumar K $P^{a}$, Sivakumar $P^{b}$ and MohanasundariL ${ }^{c}$

\begin{tabular}{|l|c|c|c|c|c|}
\hline \hline 6 & 98.8 & 98.6 & 99.8 & 98.2 & 98.1 \\
\hline 7 & 98.5 & 98.4 & 98.5 & 98.6 & 98.5 \\
\hline 8 & 97.9 & 97.9 & 99.6 & 98.8 & 98.9 \\
\hline 9 & 98.4 & 98.8 & 99.4 & 97.3 & 95.8 \\
\hline 10 & 98.8 & 98.6 & 98.9 & 97.9 & 97.1 \\
\hline Average & $\mathbf{9 8 . 3}$ & $\mathbf{9 8 . 5 6}$ & $\mathbf{9 9 . 1}$ & $\mathbf{9 8 . 1 4}$ & $\mathbf{9 8 . 2}$ \\
\hline
\end{tabular}

Fig. 6 is the pictorial analysis of haze image classification approach on indoor environmental images.

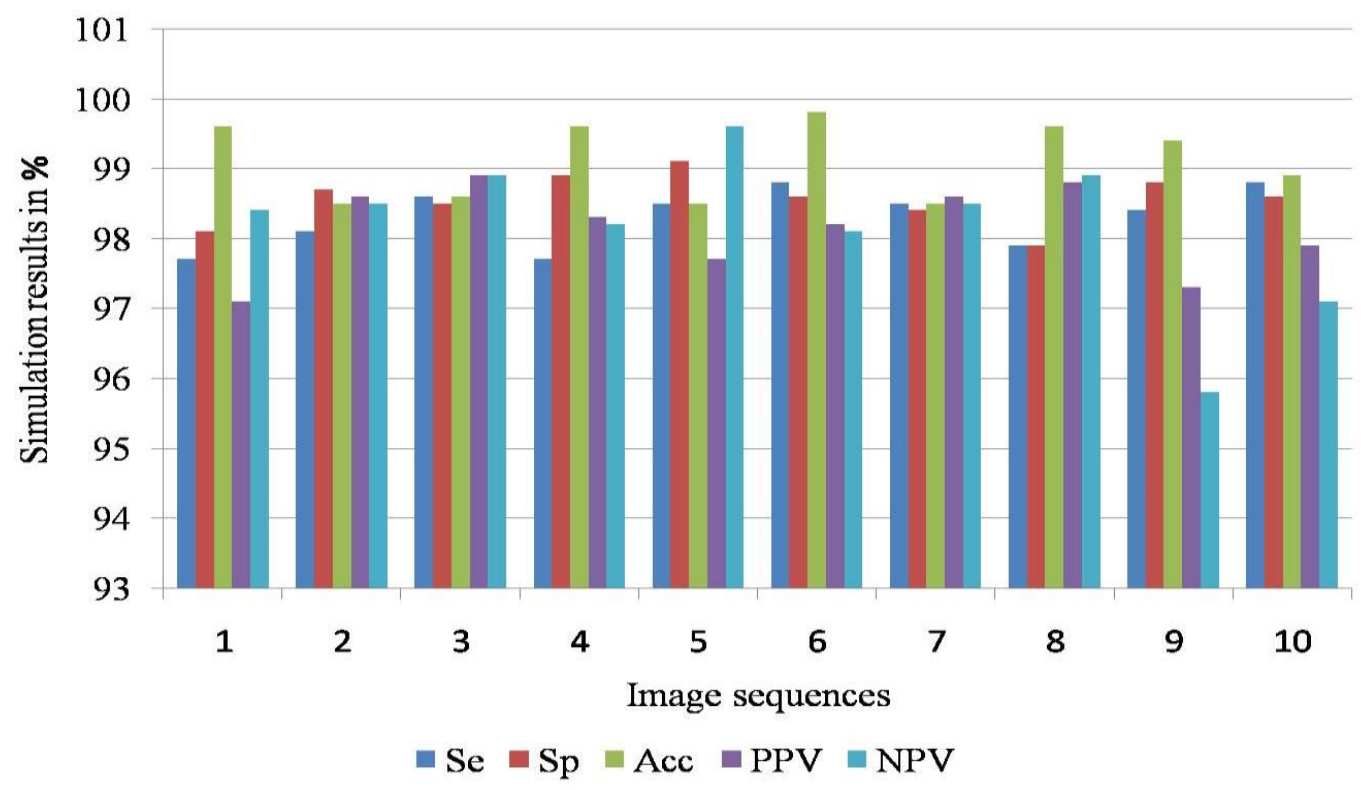

Figure 6 Pictorial analysis of haze image classification approach on indoor environmental images

Table 2 is the simulation results analysis of the proposed haze image classifications on Outdoor images. The proposed haze image classification system obtains $96.99 \%$ of sensitivity, $98 \%$ of specificity, and $98.19 \%$ of accuracy, $94.24 \%$ of PPV and $97.67 \%$ of NPV.

Table 2 Simulation results analysis of the proposed haze image classifications on Outdoor images

\begin{tabular}{|c|c|c|c|c|c|}
\hline \multirow{2}{*}{$\begin{array}{c}\text { Image } \\
\text { sequences }\end{array}$} & \multicolumn{5}{|c|}{ Performance evaluation parameters in \% } \\
\hline & Se & Sp & Acc & PPV & NPV \\
\hline 1 & 96.7 & 97.1 & 97.7 & 89.6 & 98.1 \\
\hline 2 & 96.9 & 98.5 & 98.1 & 88.1 & 97.6 \\
\hline 3 & 97.1 & 98.4 & 97.5 & 87.9 & 96.9 \\
\hline 4 & 97.5 & 98.6 & 97.9 & 92.3 & 96.1 \\
\hline 5 & 96.3 & 97.5 & 97.1 & 95.8 & 97.5 \\
\hline 6 & 96.2 & 98.1 & 98.6 & 97.1 & 98.8 \\
\hline 7 & 96.8 & 97.4 & 98.3 & 98.2 & 97.1 \\
\hline 8 & 97.6 & 98.6 & 99.1 & 97.7 & 97.3 \\
\hline 9 & 97.6 & 97.7 & 98.7 & 98.1 & 98.6 \\
\hline 10 & 97.2 & 98.1 & 98.9 & 97.6 & 98.7 \\
\hline Average & 96.99 & 98 & 98.19 & 94.24 & 97.67 \\
\hline
\end{tabular}

Fig. 7 is the pictorial analysis of haze image classification approach on outdoor environmental images. 


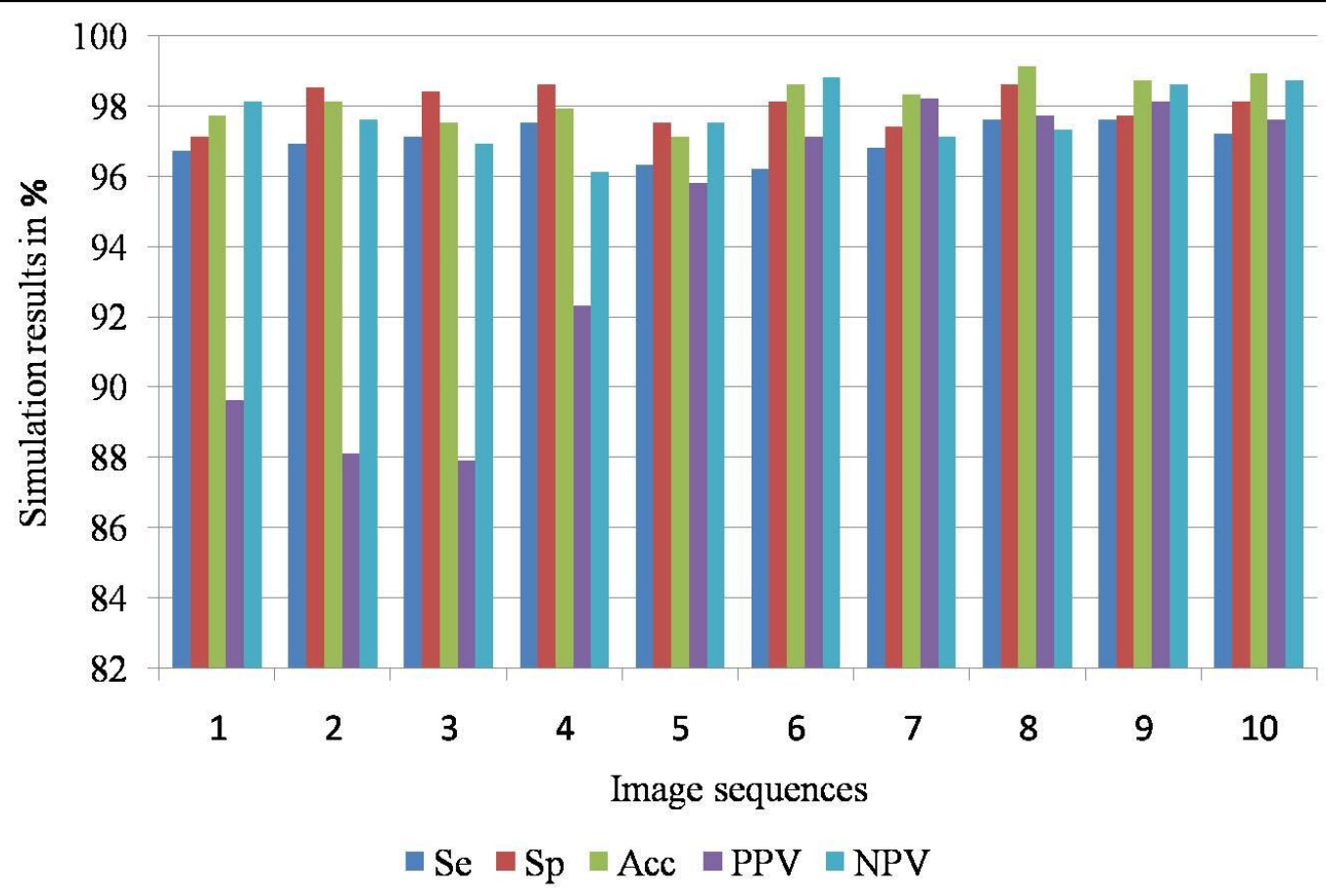

Figure 7 Pictorial analysis of haze image classification approach on outdoor environmental images

Table 3 is the analysis of the proposed haze image classification method on Indoor and Outdoor environmental images. It is analyzed that the proposed haze image classification method provides superior simulation results on indoor images than the outdoor environmental images.

Table 3 Analysis of the proposed haze image classification method on Indoor and Outdoor environmental images

\begin{tabular}{|c|l|l|l|l|l|}
\hline Environment & $\begin{array}{l}\text { Se } \\
(\boldsymbol{\%})\end{array}$ & $\begin{array}{l}\text { Sp } \\
(\boldsymbol{\%})\end{array}$ & $\begin{array}{l}\text { Acc } \\
(\boldsymbol{\%})\end{array}$ & $\begin{array}{l}\text { PPV } \\
(\boldsymbol{\%})\end{array}$ & $\begin{array}{l}\text { NPV } \\
(\boldsymbol{\%})\end{array}$ \\
\hline Indoor case & 98.3 & 98.56 & 99.1 & 98.14 & 98.2 \\
\hline Outdoor case & 96.99 & 98 & 98.19 & 94.24 & 97.67 \\
\hline
\end{tabular}

Fig. 8 is the pictorial analysis of the proposed haze image classification method on Indoor and Outdoor environmental images.

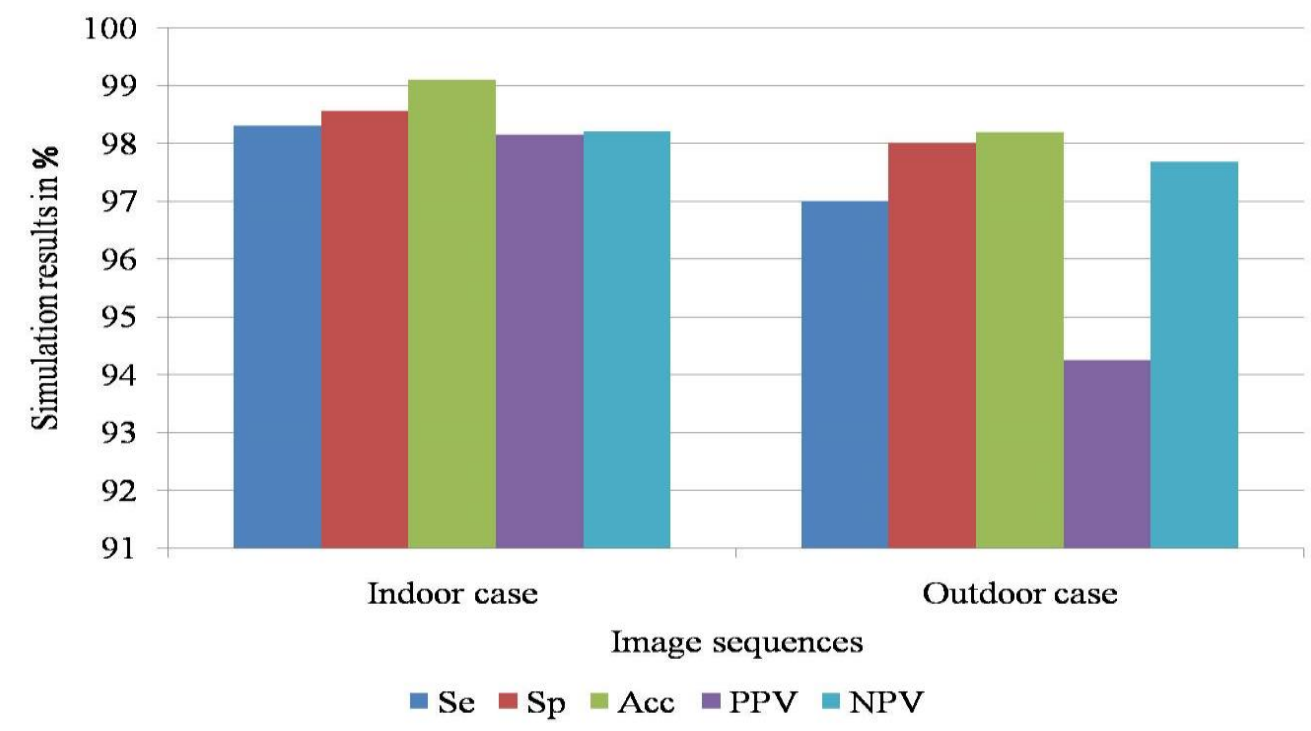

Figure 8 Pictorial analysis of the proposed haze image classification method on Indoor and Outdoor environmental images 
Table 4 is the comparative analysis of the proposed haze image classification method and conventional methods on Indoor environmental images. The proposed method for haze image classification is compared with respect to the conventional methods Hassan et al. (2020),Kang et al. (2020) and Guo et al. (2019), on indoor set of images.

Table 4 Comparative analysis of the proposed haze image classification method and conventional methods on Indoor environmental images

\begin{tabular}{|l|c|c|c|}
\hline Authors & $\begin{array}{l}\text { Se } \\
(\boldsymbol{\%})\end{array}$ & $\begin{array}{l}\text { Sp } \\
(\boldsymbol{\%})\end{array}$ & $\begin{array}{l}\text { Acc } \\
(\boldsymbol{\%})\end{array}$ \\
\hline Proposed method & 98.3 & 98.56 & 99.1 \\
\hline Hassan et al. (2020) & 97.1 & 97.6 & 97.6 \\
\hline Kang et al. (2020) & 96.5 & 97.1 & 97.2 \\
\hline Guo et al. (2019) & 96.1 & 98.1 & 98.3 \\
\hline
\end{tabular}

The pictorial comparative analysis on Indoor environmental images is shown in Fig 9.

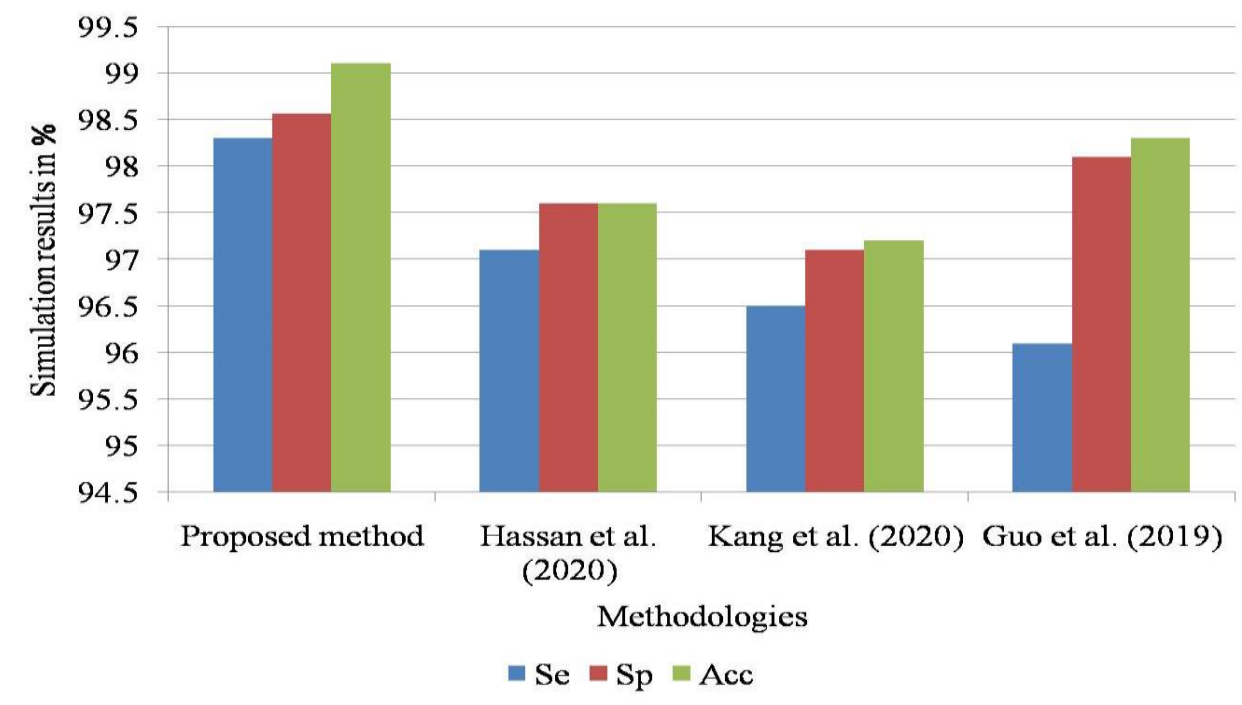

Figure 9 Pictorial comparative analyses on Indoor environmental images

Table 5 is the comparative analysis of the proposed haze image classification method and conventional methods on outdoor environmental images. The proposed method for haze image classification is compared with respect to the conventional methods Hassan et al. (2020), Kang et al. (2020) and Guo et al. (2019), on outdoor set of images.

Table 5Comparative analysis of the proposed haze image classification method and conventional methods on Outdoor environmental images

\begin{tabular}{|l|l|l|l|}
\hline Authors & $\begin{array}{l}\text { Se } \\
(\boldsymbol{\%})\end{array}$ & $\begin{array}{l}\text { Sp } \\
(\boldsymbol{\%})\end{array}$ & $\begin{array}{l}\text { Acc } \\
\mathbf{( \% )}\end{array}$ \\
\hline Proposed method & 96.99 & 98 & 98.19 \\
\hline Hassan et al. (2020) & 94.7 & 95.2 & 96.1 \\
\hline Kang et al. (2020) & 95.9 & 95.3 & 96.8 \\
\hline Guo et al. (2019) & 94.1 & 95.3 & 96.4 \\
\hline
\end{tabular}

The pictorial comparative analysis on Outdoor environmental images is shown in Fig 10. 


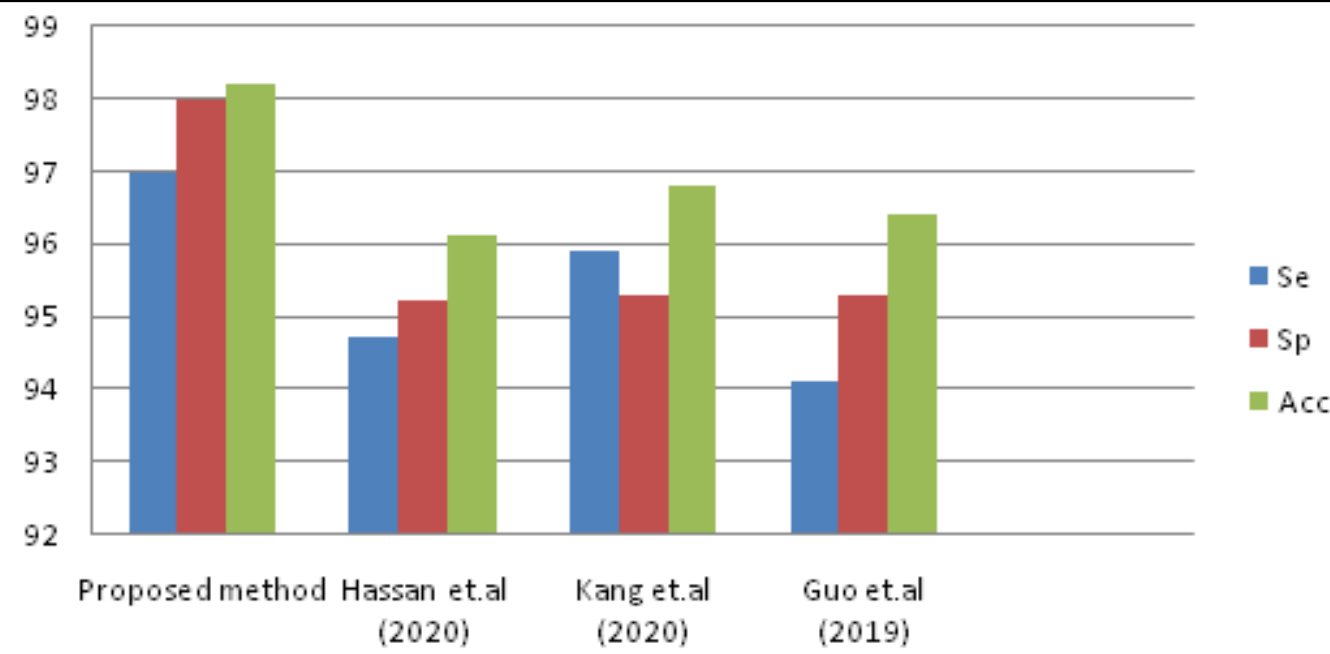

Figure 10 Pictorial comparative analyses on Outdoor environmental images

\section{Conclusions}

In this paper, deep learning classification approach based haze image detection methodology is proposed to detect the haze affected images with respect to indoor and outdoor environmental images. The proposed haze image classification system obtains $98.3 \%$ of sensitivity, $98.56 \%$ of specificity, and $99.1 \%$ of accuracy, $98.14 \%$ of PPV and $98.2 \%$ of NPV. The proposed haze image classification system obtains $96.99 \%$ of sensitivity, $98 \%$ of specificity, and $98.19 \%$ of accuracy, $94.24 \%$ of PPV and $97.67 \%$ of NPV. It is analyzed that the proposed haze image classification method provides superior simulation results on indoor images than the outdoor environmental images.

\section{References}

1. Liang Ding, Xueru Zhao, "Shearlet-Wavelet Regularized Semismooth Newton Iteration for Image Restoration", Mathematical Problems in Engineering, vol. 2015, Article ID 647254, 12 pages, 2015.

2. Gibert, X., Patel, V.M., Labate, D. et al. Discrete shearlet transform on GPU with applications in anomaly detection and denoising. EURASIP J. Adv. Signal Process. 2014, 64 (2014).

3. Hassan, H., Bashir, A.K., Ahmad, M. et al. Real-time image dehazing by superpixels segmentation and guidance filter. J Real-Time Image Proc, vol.1, 2020, pp.1-10.

4. Ju, M., Ding, C., Guo, Y.J., Zhang, D.: Remote sensing image haze removal using gamma-correction-based dehazing model. IEEE Access 7, 5250-5261 (2018).

5. Kang, M., Jung, M. A single image dehazing model using total variation and inter-channel correlation. MultidimSyst Sign Process 31, 431-464 (2020).

6. Guo, Y., Zhang, Z., Yuan, H. et al. Single Remote-Sensing Image Dehazing in HSI Color Space. J. Korean Phys. Soc. 74, 779-784 (2019).

7. Tian, Y., Xiao, C., Chen, X., Yang, D., Chen, Z.: Haze removal of single remote sensing image by combining dark channel prior with superpixel. Electron. Imaging 2016(2), 1-6 (2016)

8. Kim, J.-H., Jang, W.-D., Sim, J.-Y., Kim, C.-S.: Optimized contrast enhancement for real-time image and video dehazing. J. Vis. Commun. Image Represent. 24(3), 410-425 (2013).

9. Huang, S.-C.: An advanced motion detection algorithm with video quality analysis for video surveillance systems. IEEE Trans. Circuits Syst. Video Technol. 21(1), 1-14 (2010)

10. Jia, Z., Wang, H., Caballero, R.E., Xiong, Z., Zhao, J., Finn, A.: A two-step approach to see-through bad weather for surveillance video quality enhancement. Mach. Vis. Appl. 23(6), 1059-1082, (2012)

11. Wang, W., Yuan, X.: Recent advances in image dehazing. IEEE/ CAA J. Autom. Sin. 4(3), 410-436 (2017)

12. Jiang, H., Lu, N.: Multi-scale residual convolutional neural network for haze removal of remote sensing images. Remote Sens. 10(6), 945 (2018).

13. Meng, G., Wang, Y., Duan, J., Xiang, S., Pan, C.: Efficient image dehazing with boundary constraint and contextual regularization. In: Proceedings of the IEEE International Conference on Computer Vision, pp. 617-624.

14. Wang, Z., Feng, Y.: Fast single haze image enhancement. Comput. Electr. Eng. 40(3), 785-795 (2014).

15. Sun, L., Latifovic, R., Pouliot, D.: Haze removal based on a fully automated and improved haze optimized transformation for landsat imagery over land. Remote Sens. 9(10), 972 (2017). 